\title{
An Early Prediction of Cardiac Risk using Augmentation Index Developed based on a Comparative Study
}

\author{
Manimegalai.P \\ Assistant Professor \\ Department of Electronics and \\ Instrumentation \\ Karunya University \\ ,Coimbatore.
}

\author{
Delpha Jacob \\ Post Graduate Student, \\ Biomedical Instrumentation \\ Engineering, \\ Karunya University ,Coimbatore
}

\author{
K. Thanushkodi \\ Phd,The Director \\ Akshaya College of \\ Engineering, Coimbatore
}

\begin{abstract}
It is important to determine the cardiac risk of a patient in advance to prevent premature death. This paper gives a brief of the methods used so far to determine the cardiac risk of a patient from a PPG signal. It also compares the effectiveness of each article in various criteria including cost and the paper also presents a novel technique to determine the cardiac risk of a patient at reduced cost. The paper states to find the cardiac risk of a patient form a Photoplethysmographic Signal. Arterial stiffness leads to cardiac disorders, the degree of arterial stiffness can be obtained by calculating the augmentation index of a pulse wave. Augmentation Index is an important factor of cardio vascular risk. Augmentation Index is the measure of Arterial stiffness derived from the ascending aortic waveform. Thus by calculating the augmentation index the degree of arterial stiffness can be calculated by which cardiac risk to the patient can be diagnosed. The augmentation index is determined by implementing an algorithm.
\end{abstract}

\section{Keywords}

Augmentation Index, Cardiac Risk, Early prediction, Photoplethysmograph, Real-Time.

\section{INTRODUCTION}

It has been statistically analyzed that $75 \%$ of diabetic patients die prematurely due to cardiovascular disease (CVD). The absolute risk of cardiovascular disease in patients with type 1 (insulin-dependent) diabetes is lower than that in patients with type 2(non-insulin-dependent) diabetes. Unfortunately, about 9 out of 10 people with diabetes have type 2 diabetes. Thereby it is important to determine the cardiac risk of a patient in advance to prevent premature death [2]. The analysis of peripheral blood volume pulse helps to understand arterial pathologies, a major contributor to cardiovascular diseases, which is a common cause of death in modern society. The risk factors for cardiovascular diseases are associated with the increasing stiffness of the arterial wall. Photoplethysmograph is a measure of the volume of the blood in the vessel. Thus by analysing this peripheral pulse wave we can predict cardiovascular risk. High pulse pressure is now recognized as an important cardiovascular disease risk factor for myocardial infarction, stroke, and development and progression of heart failure, even after adjusting for the effects of other known cardiovascular disease risk factors. It is also evident that Elevated pulse pressure in elderly and hypertensive individuals is attributable to increased aortic stiffness and wave reflection [6]. Kidney transplant patients are prone to heart risks thereby a prior indication of the same can prevent premature death, Evidence demonstrates that cardiovascular risk reduces after kidney transplantation, but is still a major cause of death. With increasing inclusion of diabetic patients for kidney transplantation, the evaluation of cardiovascular disease in this population becomes more important [2]. Increased arterial wave reflections are associated with the presence and extent of coronary atherosclerosis and patients with cardiovascular mortality. Studies have reported an association between arterial function indices and cardiovascular risk factors, as well as the risk of incident cardiovascular events, including coronary heart disease and stroke [5].

\section{CONVENTIONAL APPROACHES FOR DETECTION OF CARDIAC RISK FROM PPG SIGNAL.}

2. 1. Augmentation index (AIx) is traditionally obtained from pressure waveforms via arterial applanation tonometry. Eshan Patvardhan, Kevin S [1] sought to evaluate the association between AIx obtained from peripheral arterial tonometry (PAT) with cardiovascular risk factors (CRF) and coronary artery disease (CAD). The study evaluated the association of peripheral AIx and cardiovascular risk factors. PAT-derived AIx correlates with age, weight, BMI, heart rate, diastolic blood pressure, pulse pressure, and mean arterial pressure. PAT-AIx was also significantly related to age.Various studies have noted that the increase in AIx with age is not linear. AIx values increased with age up to 65-70 years of age. Beyond that, the Aix Values tended to plateau and then decrease shortly after that. It has been observed that AIx measured by conventional applanation tonometry begins to plateau at the age of 60 years. Patients with $>5$ CRFs had a significantly higher PAT-AIx as compared with those having only a few CRFs. It suggests that finger-derived AIx may be comparable to applanation tonometry for measurement of augmentation index and evaluation of cardiovascular risk. [1] But only Patients with >5 CRFs(cardiac Risk factors) had a significantly higher PAT-AIx as compared with those having only a few CRFs, this restricts its usage. The usual charge for this testing itself is about $\$ 250$ per test

2. 2. Evidence demonstrates that cardiovascular risk reduces after kidney transplantation, but is still a major cause of death. With increasing inclusion of diabetic patients for kidney transplantation, the evaluation of cardiovascular disease in this population becomes more important. Ali $\mathrm{R}$ Khoshdel, Shane L Carney [2] and team mates compared arterial stiffness and pulse wave reflection as well as other cardiovascular risk factors in kidney transplant patients with 
and without diabetes mellitus. Augmentation index was Measured using 'SphygmoCor, version 7.1, AtCor Medical'. One hundred kidney transplant recipients, including 33 diabetic patients, were evaluated for their renal cardiovascular risk factors, including blood pressure, lipids, glucose control, homocysteine, and arterial stiffness indexes. The tests were repeated after 1 year in 47 individuals There was no significant difference in pulse wave velocity (PWV) between the diabetic and non diabetic groups, despite a greater augmentation index (AI) in the diabetic group.The novel finding of this study was the higher adjusted augmentation index in diabetic compared to non diabetic kidney transplant patients. Arterial evaluation facilitates CV risk assessment in kidney transplant patients. Kidney transplant patients with Diabetes Mellitus had comparable PWVs, but significantly greater AIs than their non-Diabetes Mellitus counterparts.[2] 2.3. This topical review by Alberto P Avolio [3] have assessed the techniques of pressure measurement that relate to the use of the cuff sphygmomanometer and to the non-invasive analysis of the peripheral and central arterial pressure waveform. Improved assessment of cardiovascular function in relation to treatment and management of high blood pressure will result from future developments in the indirect measurement of arterial blood pressure that involve the conventional cuff sphygmomanometer with the addition of information derived from the peripheral arterial pulse.

The CVProfilor device was used to analyze the pulse wave. Diagnostic Applanation Tonometry (Specaway) uses the following formula for calculating Augmentation index.

\section{$\mathbf{A I x}=(\mathbf{P s}-\mathbf{P i}) /(\mathbf{P s}-\mathbf{P d})$}

Ps- systolic pressure point, Pi-point of inflection, Pd-diastolic pressure point

Combination of sphygmomanometer and pulse waveform analysis, characterizes the cardiovascular function and stratification of cardiovascular risk. [3]

2.4. Satoru Sakuragia [4] outlines methods for the measurement of arterial stiffness, describe the physiological mechanisms that utilises arterial stiffness as an integrative marker of cardiovascular disease, and detail the evidence examining the value of arterial stiffness for prediction of adverse cardiovascular events and mortality.

Arterial stiffness can be assessed noninvasively using the following methods

i. relating change in vessel size (diameter or area) to distending pressure

ii. estimation of pulse wave velocity

iii. pulse waveform analysis.

The first method mentioned above uses echo tracking techniques. MRI is used to measure the vascular

Distensibility and compliance, etc .It can be done only on superficial arteries.

PWV(Pulse Wave Velocity) is calculated using Moens Korteweg equation :(PWV $)^{\wedge} 2=\left(\mathrm{E}^{*} \mathrm{~h}\right) /(2 \mathrm{rp})$,

Where, $\mathrm{E} \rightarrow$ slope of stress strain relationship of a given vessel, $\mathrm{P} \rightarrow$ density of fluid, $\mathrm{h} / 2 \mathrm{r} \rightarrow$ wall thickness /diameter, The third method is PWA it is performed using applanation tonometry, which uses the following formula $\mathrm{AI} \%=\mathrm{AP} / \mathrm{PP}$ $\mathrm{AP} \rightarrow$ Augmented pressure- difference between the first and the second systolic peak ,PP $\rightarrow$ normal pulse pressure (difference between the systolic blood pressure and diastolic blood pressure) Various non-invasive indices of arterial stiffness are available, [4]

The following are the limitations .Method (i) and (ii) are complicated to perform and its very costly to use MRI in the procedure. Thereby method (iii) is the comparatively better. It is clear that PWA is one of the best approaches to measure arterial stiffness
2.5. Augmentation index predict all cause mortality and cardio vascular events in coronary and end stage renal disease patients . Sophia zoungas [5] has stated two methods that are being used namely pulse wave velocity and augmentation index calculation

i. $\quad \mathrm{PWV}=\mathrm{D} / \Delta \mathrm{t}(\mathrm{m} / \mathrm{s}) \quad$ where, $\mathrm{D} \quad \rightarrow$ distance travelled in meters, $\Delta \mathrm{t} \rightarrow$ time interval in seconds.

ii. $\quad \mathbf{A I x} \%=\Delta \mathrm{P} * 100 / \mathrm{PP} \quad$ where,$\Delta \mathrm{P} \rightarrow$ (difference between shoulder of the wave and peak systolic pressure), PP $\rightarrow$ Pulse Pressure.

iii. $\quad \mathrm{SAC}($ systemic arterial compliance $)=\mathrm{Ad} / \mathrm{R}(\mathrm{Pes}-$ Pd) where, Ad $\rightarrow$ area under the diastolic portion of the pulse pressure contour, $\mathrm{R} \rightarrow$ total peripheral resistance calculated as mean arterial blood pressure/mean blood flow, Pes/Pd $\rightarrow$ endsystolic /diastolic aortic blood pressure

Corotid artery AIx increases with increase in cardiovascular risk. Radial artery AIx increases with increases coronary artery diseases. Systemic arterial compliance may be a useful for studies of patients with known coronary disease[5]

2.6. Augmentation index (AI), a measure of enhanced wave reflection, has been proposed as a bedside measure of aortic stiffness. However AI is potentially sensitive to various factorsthe effect of two drugs Omapatarilat ,Enalapril on AI was studied by Gary F. Mitchell [6]

Drugs taken :Omapatarilat (vasopeptidase inhibitor, $80 \mathrm{mg}, 75$ nos),Enalapril (converting enzyme inhibitor, $40 \mathrm{mg}, 84 \mathrm{nos}$ ) The following was calculated on carotid waveform,

i. $\quad \mathrm{Zc}$ (characteristic impedance) $\mathrm{Zc}=$ carotid pressure/ aortic flow

ii. $\quad$ SEP(systolic ejection period)

iii. Timing of wave reflection

iv. $\mathrm{AI}($ augmentation index)

Both drugs ,Had no effect on Zc ,Reduced AI, Had no effect on the reflection time, Increases the heart rate, Shortened SEP, Peripheral resistance. Thus AI depends on the amplitude of the reflected pressure wave and not the time period. And thereby it can be used to study the activity of the drug.[6]

2.7.Increased arterial stiffness, determined non invasively, has been shown to predict a higher risk of coronary atherosclerosis. Thomas Weber (7) Used 'sphygmo Cro System(At cor medical )' for his study the formula used is

$\mathrm{AIx}=\mathrm{AP} / \mathrm{PP}$, where AP -augmentation pressure( Difference between the first and the second Systolic peak), PP- pulse pressure .(SBP-DBP).

Increase in $\mathrm{AI}$ is proportional to the increase in $\mathrm{CAD}$

(coronary artery disesse). [7]

2.8. Arterial stiffness is a precursor to premature cardiovascular disease. The augmentation index (AI) and pulse pressure (PP) are cardiovascular risk factors. The aim of this study by Jin Wook Chung and team [8] was to define the diagnostic values of the $\mathrm{AI}$ and $\mathrm{PP}$ from the peripheral arterial and central aortic waveforms in healthy subjects.[8] It was understood that there was variation in Augmentation index with respect to age and other factors like obesity etc,

2.9.Arterial stiffness is an important factor for cardiovascular risk .Aix (augmentation index )is a measure of systemic arterial stiffness derived from the ascending aortic pressure waveform. The aim of Dirk Sommermeyer [9] is to analyze the relationship between the augmentation index derived from peripheral pulse wave measured by PPG(Photo plethysmography) and the two indices measured from an established device from Tensiomed Arterioraph

Formula used: AIx_amp $\%=$ P2 $* 100 / P 1$, AIx_time $\%=$ $\mathrm{T} 1 * 100 / \mathrm{T} 1$, AIx_height $\%=$ body-height $* 100 / \mathrm{T} 1$ as given in Fig. 1 
PPG appears to provide an accurate method to determine the arterial stiffness, which is an important marker for arthrosclerosis and prediction of cardiovascular risk . Henceforth the paper states that Photoplethysmography is suited for monitoring cardiac risk and it gives appropriate details required to calculate the augmentation index.[9]

Remark: In all the articles mentioned above, instruments were used to measure the augmentation index and thereby they are not cost efficient. And the methodology is tedious.

\section{PROPOSED WORK}

The novel algorithm aims at helping the patients to predict their cardiac risk at an early stage with a reduced cost. PPG (Photoplethysmograph) is one of the most vital bio measurements in an emergency care unit. This study helps in analyzing those signals to assess the cardiac risk of the patient without any aid of an additional instrument. The proposed algorithm can be used in real time to continuously monitor the cardiac state of the patient. Advantages are as follows,

i. Very simple, low cost, easy to apply PPG technique.

ii. Long term monitoring

iii. Therapy control or prevention of cardiovascular diseases. [17]

\subsection{Methodology}

The experimental set up for acquisition of pulse wave and calculating its augmentation index is given in fig. 1 PPG signals are taken from individual persons using Photoplethysmography unit with a sampling rate of 1000 samples/second. The frequency response for PPG it is 0.05$10 \mathrm{~Hz}$. It is obtained by using reflection type PPG sensor, these signal are amplified using a PPG amplifier and are interfaced with the PC using DAQ (NI USB-6009), The NI USB-6009 is a USB (Universal Serial Bus) based data acquisition (DAQ) and control device with analog input and output and digital input and output.

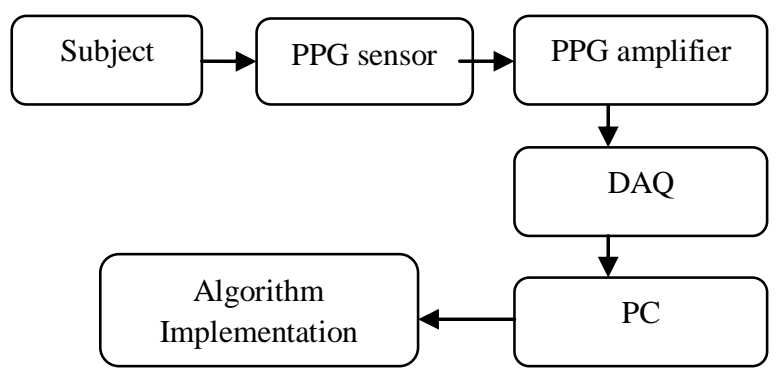

Fig 1: Block Diagram of the proposed work

The signals are then acquired using LabVIEW software and analysis is done by implementing the algorithm in MATLAB. LabVIEW aids in real time implementation. The block diagram as shown in Fig.2, the acquisition of the required PPG signal from normal and abnormal subjects were obtained

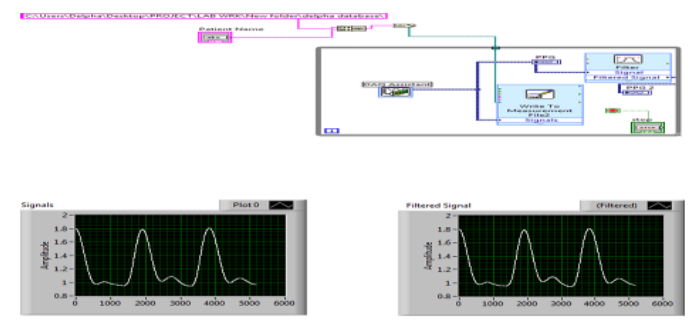

Fig 2: Acquisition of signals using LabVIEW software.
The flow chart that gives a brief account of the steps involved in calculating the augmentation index is as given in the flow chart.Fig.3 The formula [1] for calculating the augmentation index is as given below,

$$
\mathrm{Ai}=\frac{(P s-P i)}{(P s-P d)}
$$

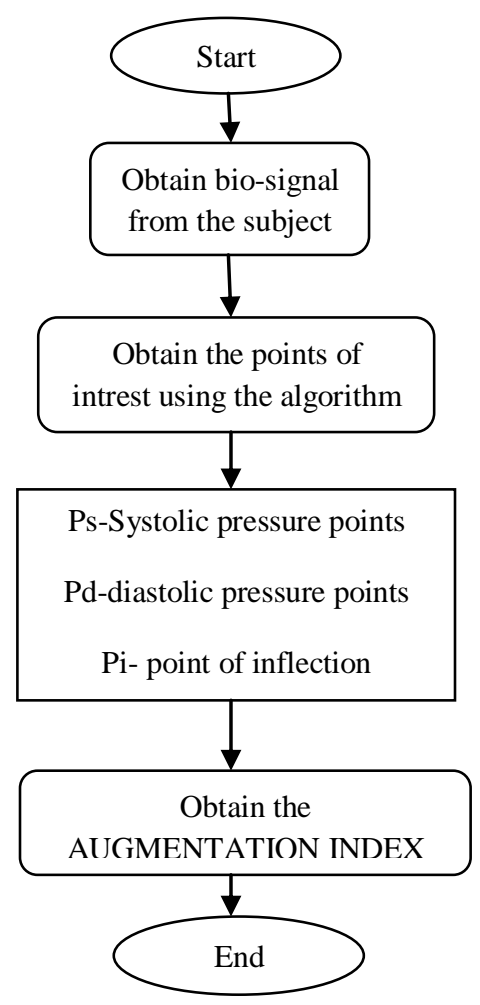

Fig 3: Flow Chart showing the steps involved in calculating AI.

\section{Discussion}

The normal and the abnormal waveforms were different in pattern. The point of inflection was present in few abnormal signals and was unpredictable in most of the abnormal signals. This appearance of the wave in mainly due to the damping effect, and thereby the point of inflection becomes difficult to detect. This is mainly due to aging, diabetes, atherosclerosis, arterial stiffness. This work was first started by assuming Pi to be zero, but later found it to be incorrect and corrected it by the following method, the second derivative of the signal is taken to detect the point of inflection. The point at which the second derivative crosses zero denotes the point of inflection. The first derivative becomes maximum at the point of inflection.[22]

\subsection{Algorithm Implementation}

\subsubsection{Calculating the extremas:}

The following is the concept of calculating the extrema of the given signal. The steps involved are as follows,

(i) To find Local maximas:

$x=a$ is a point of maxima value of $f$, if 
- $\mathrm{f}(\mathrm{a})=0$ and,

- $f(x)$ changes sign from positive to negative as $x$ increases through a, i.e. $f(x)>0$ at every point sufficiently close to and to the left of $a$, and $f(x)<0$ at every point sufficiently close to and to the right of a.

- Therefore the gradient is decreasing.

(ii) To find Global maxima:

The maximum value among the local maximas is given as the global maxima.

(iii) To find the Local minimas:

$\mathrm{x}=\mathrm{a}$ is a point of minima value of $\mathrm{f}$, if

- $\mathrm{f}(\mathrm{a})=0$ and,

- $\quad f(x)$ changes sign from negative to positive as $x$ increases through a, i.e. $\mathrm{f}(\mathrm{x})<0$ at every point sufficiently close to and to the left of a, and $f(x)>0$ at every point sufficiently close to and to the right of $\mathrm{a}$.

- Therefore the gradient is increasing.

(iv) To find the Global minima:

The maximum value among the local minimas is given as the global minima.[17]

\subsubsection{Identify the type of signal:}

The signal has to be studied in advance so as to indentify if the point of Inflection is noticeable or not. If the point of inflection is present then the value can be taken and directly substituted in the formula (1). If the point of inflection is unnoticeable then the derivative of the signal is taken and the point of inflection is found using the derivative. The illustration of this method is shown clearly in Figure 8.

\subsubsection{Calculating single cycle:}

The maxima is detected, for every peak, a minimum point to the left and two minimum point to the right is calculated , and this comprises a single cycle. For each cycle the Augmentation Index calculated and the average is taken .

\subsubsection{Calculating the augmentation index:}

The formula used to calculate Augmentation Index is given as follows.

$$
\mathrm{Ai}=\frac{\mathrm{P}_{s}-\mathrm{Pi}_{\mathrm{i}}}{\mathrm{P}_{s}-\mathrm{Pd}}
$$

Where,

Ps $\rightarrow$ systolic pressure point.

Pd $\rightarrow$ diastolic pressure point

$\mathrm{Pi} \rightarrow$ point of inflection.

Using the above equation the augmentation index wa calculated.[17]

\section{Subjects:}

The normal subjects where within the age of 40 . The signals were obtained for about a min after the subject completely relaxes. The abnormal subject were with various degrees of cardiac risk , the abnormal signals were taken from patients admitted for complications that lead to cardiac risk like diabetes, bradycardia etc. there were above the age of 40 .

\section{RESULTS}

\subsection{MATLAB results}

The following were the results obtained by implementing the above steps of the algorithm to calculate the augmentation index of the acquired signal.

The implementation of the steps in MATLAB is given as below, The resuls given below is for healthy subject where the point of inflection is present. Pi mostly present in all healthy subjects.
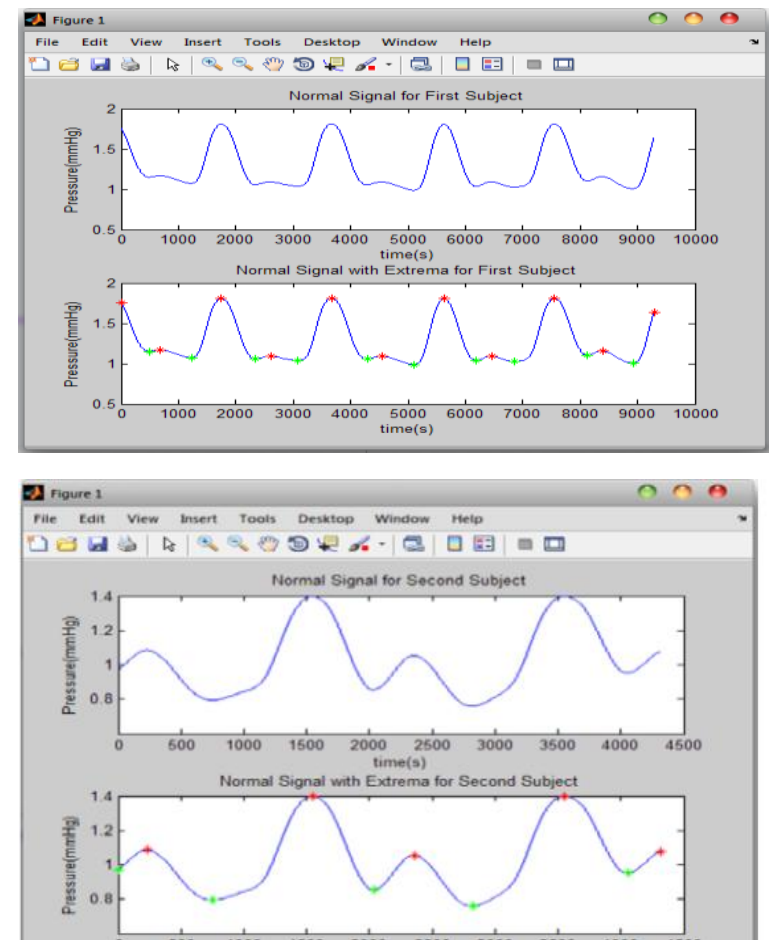

(a) First Subject

(b) Second Subject

Fig 4: Normal subjects
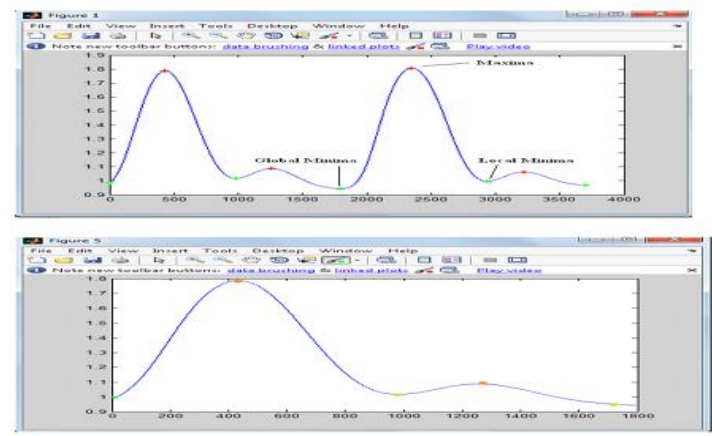

Fig 5: Finding maximas and minimas and extracting a single cycle. 


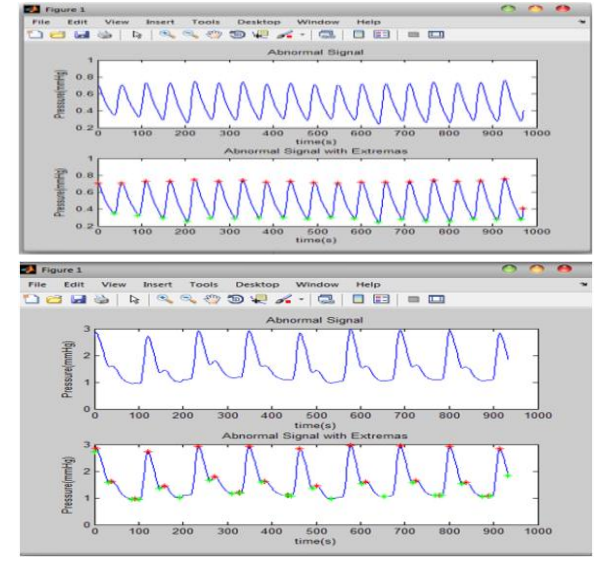

(a) First subject

(b) Second subject

Fig 6: Abnormal Subject

In Fig.6, the abnormal signal with Pi and abnormal signal without Pi is shown. in most abnormal cases the PPG signal obtained will have significant Pi. When Pi is not seen in the obtained signal, as mentioned earlier the derivatives are taken to locate the Pi point. The following Fig. 8 illustrates this concept.

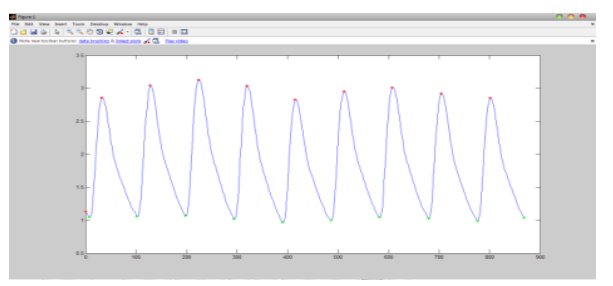

Fig 7: Abnormal signal with no Pi

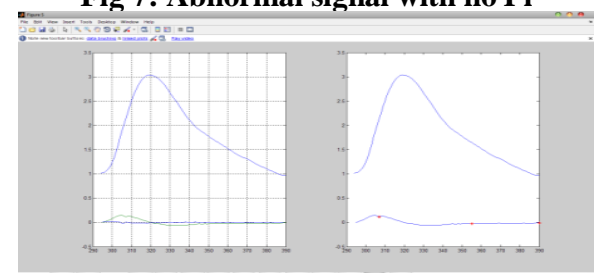

Fig 8: Illustration of locating the $\mathrm{Pi}$. a) Shows the original signal with its first and second derivative

b). Shows the location of the $\mathrm{Pi}$ on the original signal with respect to its derivative

4.2 LabVIEW results.

Thus by the above procedures the Augmentaion index was calculated for abnormal and normal signals. The same was implemented using LabVIEW and the results are as follows



Fig 9: Calculation of AI (Augmentation Index )for signals with Pi (Point of Inflection)

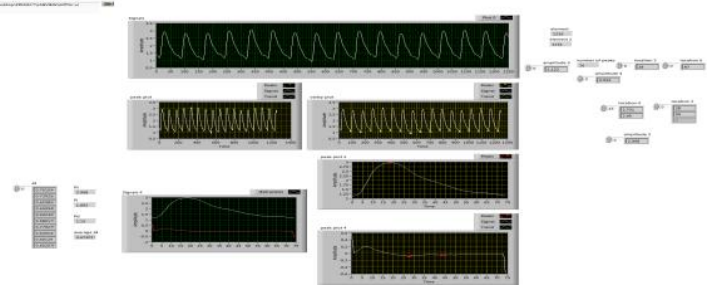

Fig10: Calculation of AI (Augmentation Index )for signals with no Pi (Point of Inflection)

The following is the table stating the augmentation index of normal and abnormal subjects. A clear variation can be seen in the value of $\mathrm{AI}$.

\section{TABLE 1. TABLE OF COMPARISON OF ALL DATA OBTAINED FROM HEALTHY AND ABNORMAL SUBJECT:}

\begin{tabular}{|c|c|c|c|c|}
\hline Subject & Ps & Pi & Pd & AI \\
\hline \multicolumn{5}{|c|}{ Normal Subject } \\
\hline Patient 1 & 0.099426 & 0.97848 & 0.97234 & 0.719725 \\
\hline Patient 2 & 0.108701 & 0.10141 & 0.10108 & 0.956921 \\
\hline Patient 3 & 0.102981 & 0.9486 & 0.94228 & 0.933464 \\
\hline Patient 4 & 0.102878 & 0.09895 & 0.09836 & 0.868795 \\
\hline Patient 5 & 0.133413 & 0.12499 & 0.12360 & 0.858548 \\
\hline \multicolumn{5}{|c|}{ Abnormal Subject } \\
\hline Patient 1 & 2.9830 & 2.3810 & 1.1480 & 0.2932 \\
\hline Patient 2 & 2.9070 & 1.8360 & 1.1890 & 0.5776 \\
\hline Patient 3 & 2.9520 & 1.8710 & 0.9960 & 0.5945 \\
\hline Patient 4 & 2.5960 & 2.1120 & 1.0490 & 0.3129 \\
\hline Patient 5 & 0.7290 & 0.4030 & 0.2600 & 0.6958 \\
\hline & & & & \\
\hline
\end{tabular}

\section{Ps(Systolic Pressure Point),Pi(Point of}

Inflection),Pd(Diastolic Pressure Point), AI(Augmentation Index)

\section{CONCLUSION}

This paper states to find the cardiac risk of a patient form a Photoplethysmographic Signal. Photoplethysmography (PPG) is the electro-optic technique of measuring the cardiovascular pulse wave found throughout the human body. The pulse wave is caused by the periodic pulsations of arterial Blood volume. Arterial stiffness leads to cardiac disorders, the degree of arterial stiffness can be obtained by calculating the augmentation index of a pulse wave . Augmentation Index is an important factor of cardio vascular risk. Augmentation Index is the measure of Arterial stiffness derived from the ascending aortic waveform. Thus by calculating the augmentation index the degree of arterial stiffness can be 
calculated by which cardiac risk to the patient can be diagnosed. This paper includes the literature survey of the same, in which the key points are summed up below every division. In almost all the paper studies so far every study uses tonometry and hence it's not cost effective, this paper states an algorithm which can replace the use of any instrument and hence becomes cost effective and most efficient. It can be used along with the normal bed side patient monitor to continuously monitor the cardiac state of the patient.

\section{REFERENCES}

[1] Eshan Patvardhan, Kevin S. Heffernan, Jenny Ruan,Michael Hession, PatrickWarner, Richard H. Kara's, and Jeffrey T. Kuvin1 "Augmentation Index Derived from Peripheral Arterial Tonometry Correlates with Cardiovascular Risk Factors"Cardiology Research and Practice 2011

[2] Ali R Khoshdel, Shane L Carney, Paul Trevillian, Alastair Gillies1." Evaluation of Arterial Stiffness and Pulse Wave Reflection for Cardiovascular Risk Assessment in Diabetic and Non-diabetic Kidney Transplant Recipients", Iranian Journal of Kidney Diseases | Volume 4 | Number 3 | July 2010.

[3] Alberto P Avolio1, Mark Butlin and Andrew Walsh "Arterial blood pressure measurement and pulse wave analysis - their role in enhancing cardiovascular Assessment",Institute of Physics and Engineering in Medicine 2010

[4] Satoru Sakuragi a, Walter P. Abhayaratna "Arterial stiffness: Methods of measurement, physiologic determinants and prediction of cardiovascular outcomes”, International Journal of Cardiology 2009

[5] Sophia zoungas,Roland P.Asmar "Arterial Stiffness And Cardiovascular Outcomes" Clinical and experimental pharmacology and physiology (2007)

[6] Gary F. Mitchell, Yves Lacourcière, J. Malcolm O. Arnold, Mark E. Dunlap, Paul R. Conlin and Joseph L. Izzo, Jr "Changes in Aortic Stiffness and Augmentation Index After Acute Converting Enzyme or Vasopeptidase Inhibition", Journal of the american heart association. 2005

[7] Thomas Weber, Johann Auer, Michael F. ORourke, Erich Kvas, Elisabeth LassnigRobert Berent and Bernd Eber „Arterial Stiffness, Wave Reflections, and the Risk of Coronary Artery Disease“" Journal of the american heart association.2004

[8] Jin Wook Chung, Young Soo Lee, Jeong Hyun Kim, Myung Jun Seong, So Yeon Kim, Jin Bae Lee, Jae Kean Ryu, Ji Yong Choi, Kee Sik Kim, Sung Gug Chang, Geon Ho Lee, and Sung Hi Kim "Reference Values for the Augmentation Index and Pulse Pressure in Apparently Healthy Korean Subjects".2010 The Korean Society of Cardiology

[9] Dirk Sommermeyer, Uwe Nixdroff, Bernd Scholler, Armen Bolz "Determination Of Arterial Stifness By Photoplethysmography For Prediction Of Cardiovascular Risk".Institute Of Bio Medical Engg, Germany

[10] Mohamad E. Alnaeb, Nasser Alobaid, Alexander M. Seifalian, Dimitri P. Mikhailidis and George Hamilton
"Optical Techniques in the Assessment of Peripheral Arterial Disease", Current Vascular Pharmacology, 2007, 5, 53-59

[11] Satoru Sakuragi, Walter P. Abhayaratna "Arterial stiffness: Methods of measurement, physiologic determinants and prediction of cardiovascular outcomes", International Journal of Cardiology 138 (2010) 112-118

[12] Bronwyn A. Kingwell and Christoph D. Gatzka "Arterial stiffness and prediction of cardiovascular risk", Journal of Hypertension 2002, 20:2337-2340

[13] Juan C Grignola, Enric Domingo, Lucía Devera, Fernando Ginés "Assessment of right ventricular afterload by pressure waveform analysis in acute pulmonary hypertension", World J Cardiol 2011October 26; 3(10): 322-328 ISSN 1949

[14] Patrick Segers, Ahmad Qasem, Tine De Backer, Stephane Carlier, Pascal Verdonck Avolio "Peripheral "Oscillatory" Compliance Is Associated With Aortic Augmentation Index" , Hypertension 2001, 37:14341439

[15] Michael F. O'rourke, M.D., D.Sc., Andwilmer W. Nichols, Ph.D.* "Potential For Use Of Pulse Wave Analysis In Determining The Interaction Between Sildenafil And Glyceryl Trinitrate", Clin. Cardiol. 25, 295-299 (2002)

[16] U. Rubins, A. Grabovskis, J. Grube and I. Kukulis "Photoplethysmography Analysis of Artery Properties in Patients with Cardiovascular Diseases", NBC 2008, Proceedings 20, pp. 319-322, 2008 Springer-Verlag Berlin Heidelberg 2008

[17] Delpha Jacob ${ }^{1}$,Manimegalai Vairavan ${ }^{2}$, Dr Thanuskodi ${ }^{3}$ "Analysis of Pulse Wave to Determine the Cardiac Risk of the Patient" International journal of Advanced Scientific and Technical Research Issue 1, Vol 2 December 2011 ISSN 2249-9954

[18] http://www.equipmentexplained.com/physics/respi_meas urements/oxygen/oximeter/pulse_oximeter.html\#oxygen saturation

[19] http://terpconnect.umd.edu/ toh/spectrum/Differentiation html\#Applications

Text books refered:

[20] R.S.Khandpur, "Handbook of Biomedical Instrumentation", Second edition, Tata McGraw Hill, New York.2007.

[21] DC Reddy "Biomedical signal Processing Principles and Techniques", Tata McGraw Hill

[22] "Biophotonics And Coherent Systems In Biology"Lev Vladimirovich Belousov, V. L. Voeikov, Vladimir Leonidovich Voeǐkov, Victor S. Martynyuk

Biophotonics optical sciences and engineering for the 21 st century" by Xun Shen and Roeland Van Wijk 